\title{
Abstracts of Award-Winning Posters 10th Annual Health Sciences Poster Conference, Faculty of Medicine, Health Sciences Centre Kuwait University, Kuwait, April 25-27, 2005
}

\section{Dr. Nael A. Al-Naqeeb Award for the Best Undergraduate Student Award}

\section{1 \\ Comparison of Methods for the Assessment of Urine Concentration in Patients with Microalbuminuria - Is Urine Creatinine Our Best Choice?}

K.S. El-Hammasi ${ }^{\text {a }}$, J.M. Abbas ${ }^{\text {b }}$, K.Y. Al-Husainan ${ }^{\text {b }}$, A. Abdullah ${ }^{\mathrm{b}}$, O.A. Mojiminiyi ${ }^{\mathrm{a}, \mathrm{b}}$

aKuwait University Faculty of Medicine, ${ }^{\mathrm{b}}$ Biochemistry Labratories, Mubarak Al-Kabeer Hospital, Kuwait

Introduction and Objective: The gold standard method for assessing urine microalbumin (MA) excretion is $24 \mathrm{~h}$ urine collection, but this is tedious, expensive and often inaccurate. Most commonly used alternative is early morning urine MA which depends on the patient's state of hydration and urine concentration. Urine creatinine concentration is routinely used to correct for changes in urine concentration, but studies have shown that specific gravity (SG) could be used. However, urine osmolality is the gold standard method of estimating urine concentration. The aim of this study was to compare the use of urine osmolality and SG with the widely used creatinine for estimation of urine concentration in the assessment of microalbuminuria. Methods: Hundred and sixty-two consecutive urine specimens received for MA estimation were analysed for MA, creatinine, SG and osmolality. The MA:creatinine ratio was used to classify patients as normo- $(\mathrm{n}=125)$, micro- $(\mathrm{n}=$ $30)$ or macroalbuminuric ( $\mathrm{n}=7)$ according to guidelines. Results: Urine creatinine showed moderate but significant $(\mathrm{p}<0.0001)$ correlation with SG $\left(r^{2}=0.46\right)$ and osmolality $\left(r^{2}=0.38\right)$. However, the MA:creatinine ratio showed significant correlation with the MA:SG ratio $\left(r^{2}=0.92 ; p<0.0001\right)$ and the MA:osmolality ratio $\left(\mathrm{r}^{2}=0.92 ; \mathrm{p}<0.0001\right)$. The MA:osmolality and MA:SG ratios showed significant correlation with each other $\left(r^{2}=0.93 ; p<\right.$ 0.0001). Using linear regression analysis, the corresponding cut-off values for classification of patients as micro- and macroalbuminuric are, respectively, 0.17 and 2.2 for the MA:SG ratio and 0.26 and 6.2 for the MA:osmolality ratio. Conclusions: The poorer correlation of creatinine with urine osmolality and SG suggests that it is a poor indicator of urine concentration. Our results show that urine osmolality or SG may provide more reliable estimates of urine MA measurements, and we propose that the above cut-off values be used for classification of patients' MA status.

\section{Postgraduate Student Awards}

\author{
Basic Sciences
}

\section{1}

Mammalian Cell Entry Proteins Encoded by the mce3 Operon Are Expressed during in vitro Growth of Mycobacterium tuberculosis and Internalized by HeLa Cells

S. El-Shazly a , S. Ahmad ${ }^{\text {a }}$, A.S. Mustafa ${ }^{a}$, R. Al-Attiyah
a,
D. Krajci

${ }^{a}$ Department of Microbiology and ${ }^{b}$ Department of Anatomy and EM Unit, Faculty of Medicine, Kuwait University, Kuwait

Introduction and Objective: The mammalian cell entry 3 (mce3) is one of four mce operons of Mycobacterium tuberculosis that encodes six (Mce3A-F) invasin-like proteins possibly involved

\section{KARGER}

Fax +41613061234 E-Mail karger@karger.ch www.karger.com
(C) $2005 \mathrm{~S}$. Karger AG, Basel

$1011-7571 / 05 / 0146-0444 \$ 22.00 / 0$

Accessible online at: www.karger.com/mpp 
in macrophage entry, a process central to the pathogenesis of tuberculosis and stable persistent infection. Previous studies with proteins encoded by mce1 operon have shown that Mce1A is expressed in M. tuberculosis, and polystyrene beads coated with Mce1A are internalized by mammalian cells. This study aimed to demonstrate the expression of Mce3A-F proteins during in vitro growth of $M$. tuberculosis and their role in the internalization of this bacterium by mammalian cells. Methods: Purified recombinant Mce proteins were used to raise antibodies in rabbits. The expression of Mce3AF proteins during in vitro growth of $M$. tuberculosis was demonstrated by using fractionated subcellular protein samples and Western blotting as well as total RNA and reverse transcription PCR. Fluorescent polystyrene beads coated with Mce3 proteins were used to study their uptake by HeLa cells using flow cytometry and electron microscopy. Results: The mce3A-F mRNA were detected by reverse transcription PCR. Anti-Mce3A, anti-Mce3D and antiMce3E antibodies reacted with a protein of apparent molecular size expected for the corresponding Mce3 protein in the cell wall fraction only, prepared from in vitro-grown $M$. tuberculosis. Interestingly, the beads coated with Mce3A and Mce3E were found to be abundantly associated with HeLa cells by fluorescent microscopy and flow cytometry. Scanning and transmission electron microscopy confirmed the internalization of beads coated with Mce3A and Mce $3 \mathrm{E}$. Conclusions: The Mce $3 \mathrm{~A}-\mathrm{F}$ proteins are expressed and localized in the cell wall fraction of in vitro-grown $M$. tuberculosis. The internalization of beads coated with Mce3A/Mce3E by nonphagocytic HeLa cells indicates that Mce3 proteins may also be involved in the interaction of this pathogen with macrophages and contribute towards persistent infection.

\section{Resident}

\section{2 \\ Plasma Concentrations of C-Reactive Protein and Total Homocysteine in Relation to the Severity and Risk Factors for Cerebrovascular Disease}

M.Y. Zaki a, O.A. Mojiminiyi ${ }^{\mathrm{b}}$, N.A. Abdella $^{\mathrm{c}}$, S. George $^{\mathrm{b}}$, C. Pinto ${ }^{\mathrm{C}}$, R. Mathew ${ }^{\mathrm{b}}$

${ }^{a}$ Ministry of Health, Departments of ${ }^{b}$ Pathology and ${ }^{\mathrm{c} M e d i c i n e,}$ Faculty of Medicine, Kuwait University, Kuwait

Introduction and Objective: Higher C-reactive protein (CRP) and plasma homocysteine (tHcy) concentrations have been shown to indicate increased risk of coronary heart disease and cerebrovascular disease (CVD), but the mechanisms by which they increase the risk of atherothrombotic disease are under investigation. This study evaluates the relation of CRP and tHcy with CVD risk factors. Methods: High-sensitivity CRP (hsCRP), fasting tHcy and lipid profile were determined in 50 patients with CVD and 20 healthy control subjects. Clinical data, NIHS scale on admission and disability ranking on discharge were recorded. Cutoff points of $1.5 \mathrm{mg} / \mathrm{dl}$ (hsCRP) and $15 \mu \mathrm{mol} / \mathrm{l}$ (tHcy) were used to indicate increased risk, based on epidemiological studies. Univariate and mul- tivariate logistic regression analyses were used to relate these parameters with each other, with CVD risk factors, severity and disability on discharge. Results: Thirty-eight percent of patients had increased CRP and 26\% had elevated tHcy. CRP $(p=0.01)$ and tHcy $(\mathrm{p}<0.0001)$ concentrations were significantly higher in patients compared with controls, and these differences remained significant after correction for age and sex. tHcy showed significant correlations with hsCRP ( $r s=0.35 ; \mathrm{p}=0.003$ ) and LDL cholesterol ( $r s=0.49 ; p=0.005)$. Logistic regression analysis with CVD as the dependent variable showed significant association with CRP $(p=0.01)$ and tHcy $(p<0.0001)$ only; no associations were found with lipid parameters. CRP showed increased trend with disease severity and increased disability. Conclusions: These data support 4 main conclusions: (a) elevation of CRP and tHcy are common in CVD; (b) the significant relationship between tHcy and hsCRP suggests that the association of tHcy with CVD risk is dependent on inflammation-related mechanism; (c) increased hsCRP and tHcy show that patients with CVD may be at greater risk of subsequent coronary heart disease; (d) admission hsCRP could be used as an indicator of prognosis.

\section{Basic and Applied Sciences Awards}

\section{1 \\ Non-Invasive Detection of Survivin, a Sensitive Novel Marker in Bladder Cancer Diagnosis}

\author{
M. Al-Maghrebi ${ }^{\text {a }}$, E.O. Kehinde ${ }^{\text {b }}$, J.T. Anim ${ }^{\mathrm{c}}$, K. Kapila $^{\mathrm{c}}$, \\ V. Abraham $^{\text {b }}$, G. Varadharaj ${ }^{\mathrm{c}}$ \\ Departments of a Biochemistry, ${ }^{\mathrm{b}}$ Surgery and ${ }^{\mathrm{C} P a t h o l o g y,}$ \\ Faculty of Medicine, Kuwait University, Kuwait
}

Introduction and Objective: Novel cancer-specific markers have been identified. They may aid in early diagnosis of cancer and help to differentiate between malignant and benign growth. Survivin, an apoptosis inhibitor, is amongst the highest expressed transcripts in common cancers. The aim of this study is to describe a real-time RT-PCR assay, recently developed by us, based on TaqMan technology, for accurate and reproducible determination of survivin mRNA expression in voided urine samples of patients with various categories of transitional cell carcinoma (TCC) of the bladder. Methods: We quantified survivin gene expression in precystoScopy voided urine samples from patients with: no bladder cancer (healthy patients/control), newly diagnosed TCC of bladder, recurrent TCC of bladder and those with TCC of bladder in remission. Total RNA was purified and reversely transcribed into cDNA. Primers and TaqMan probes for the cDNA-specific realtime quantitative PCR assay were designed for survivin and GAPDH. The level of GAPDH expression was measured in all samples to normalize for sample-to-sample differences in RNA input, quality and reverse transcription efficiency. Results: Survivin was detected in the urine samples of all 4 groups. Prelimi- 
nary real-time measurement of normalized survivin expression was 17 -fold higher in urine from patients with newly diagnosed TCC than in urine from healthy patients. Urine from patients with recurrent TCC showed a 99-fold increase in survivin expression as compared with urine from healthy patients. Urine from patients with TCC in remission had below baseline levels of survivin mRNA. Conclusions: The present preliminary study demonstrates that accurate quantitative measurement of survivin expression has high potential for identification of tumor cells in urine. This noninvasive and sensitive method may prove to be of interest for molecular tumor diagnostics.

\section{2 \\ Microarray-Based Gene Expression Profiling in Diabetes: Role of ReceptorTyrosine Kinases in Vascular Dysfunction}

\author{
H. Canatan a , I.F. Benter ${ }^{\text {a }, ~ M . H . M . ~ Y o u s i f ~}{ }^{\text {a }}$, S. Akhtar ${ }^{\text {b }}$ \\ aDepartment of Pharmacology and Toxicology, \\ Kuwait University Faculty of Medicine, Kuwait; \\ ${ }^{b}$ Centre for Genome-Based Therapeutics, \\ The Welsh School of Pharmacy, Cardiff University, UK
}

Introduction and Objective: The alteration of vascular reactivity in diabetes is associated with increase in phosphorylated epidermal growth factor receptor (EGFR) that may play a key role in diabetes-induced vascular dysfunction signaling. These effects were attenuated in diabetic rats treated either with RTKs (genistein) or EGFR-specific (AG1478) inhibitors. Gene expression changes in diabetic rats and the effects of the two inhibitors were assessed using microarray gene expression profiling technology. Methods: Diabetes was induced in female Wistar rats by STZ. Four groups were studied: (1) untreated controls; (2) diabetic rats without treatment; (3 and 4) diabetic rats treated with genistein or AG1478, respectively. Total RNA was isolated from mesenteric bed tissues. The hybridization to the array slides was carried out following MWG protocol. Arrays were scanned for Cy3 and Cy5 using Affymetrix 428 Array Scanner (Affymetrix Inc., Santa Clara, Calif., USA). The microarray data analysis packages, ImaGene and GeneSight (BioDiscovery, El Segundo, Calif., USA), were used for gene expression profiling analyses. Results: Using microarray profiling on 10,000 genes/slide, we determined that 569 genes showed a change in expression, by at least twofold (302 genes were upregulated, whereas 267 genes were downregulated). Treatment with AG1478 reduced the number of genes to 436 (186 upregulated and 250 downregulated). However, treatment with genistein reduced the number of genes showing change in expression, by twofold or greater, to 168 genes (47 upregulated, 121 downregulated). Conclusions: EGFR signaling pathway is likely to play a key role on the onset of cardiovascular complications, such as the alterations in the reactivity of blood vessels, linked with diabetes. Microarray gene expression profiling showed that the number of gene expression changes induced in the mesenteric bed tissues of diabetic animals were attenuated by approximately 70 and 30\% in genistein and AG1478 in the mesenteric tissues of treated animals, respectively.

\section{Clinical Sciences Awards}

\section{1 \\ Establishment of a Multiplex PCR Assay for the Detection of Respiratory Viruses in Clinical Specimens}

\author{
S. Loutfy, D. Khalik, W. Al-Nakib \\ Department of Microbiology, Faculty of Medicine, Kuwait \\ University, Kuwait
}

Introduction and Objective: Respiratory viruses are leading causative agents of global morbidity and mortality. Respiratory syncytial virus (RSV), influenza A and B (flu A, B), parainfluenza viruses types 1,3 (PIV1, -3) and adenoviruses (ADV) contribute significantly to acute respiratory infections (ARI). Since these viruses cause similar clinical manifestations, laboratory diagnosis is essential to identify the etiological agent. We aimed at establishing a genomic detection assay for diagnosing ARI caused by the six respiratory viruses. Methods: Respiratory specimens collected from 65 patients with ARI were processed for genomic amplification. The designed multiplex PCR utilized 6 primer sets and targeted the following genes: fusion protein for RSV between nucleotides 6227 and 6466, nonstructural proteins for flu A, B (from nucleotide 467 to 656 and 732 to 977 respectively), hemagglutininneuraminidase for PIV1 (from 7372 to 7551), fusion protein for PIV3 (from 5073 to 4907) and hexon protein for ADV (from 2755 to 2888). For amplifying the targeted genes of the viruses, 2 master mixtures were used; mixture 1 contained the primers specific for flu A, B and ADV, while mixture 2 the ones specific for RSV and PIV1, -3. Results: Both of the master mixtures were tested on samples spiked with standard strains of viruses; flu A, B and ADV for Mix 1, RSV and PIV1, -3 for Mix2. This showed that all the target virus strains present in the samples could be detected by the mixtures. Specificity of the mixtures were tested on samples containing rhino-, corona-, measles- and mumps viruses. None of them was amplified by the primer mixtures. Of the 65 respiratory specimens tested with both mixtures $4(6 \%)$ were positives for each of RSV, flu A and B and 3 (4.6\%) for ADV. All were negatives for PIV1, -3. Conclusions: The two-mixture-based, multiplex RT-PCR provides a novel approach for rapid and sensitive diagnosis of 6 important respiratory viral infections. 


\section{2 \\ Enzyme-Based Parasite Lactate Dehydrogenase Test for Rapid Diagnosis of Plasmodium falciparum and Plasmodium vivax}

\author{
A. Sher ${ }^{\mathrm{a}}$, J. Iqbal ${ }^{\mathrm{b}}$, P.R. Hira ${ }^{\mathrm{b}}$, Gh.H.M. Hameed ${ }^{\mathrm{c}}$, \\ Y. Mandakar ${ }^{\mathrm{e}}$, S. Al-Mufti ${ }^{\mathrm{d}}$, R.A. Al-Owaish ${ }^{\mathrm{e}}$ \\ ${ }^{a}$ Malaria Laboratory, ${ }^{b}$ Department of Microbiology, Faculty \\ of Medicine, Kuwait University, 'Ports and Borders Health \\ Division, ${ }^{d}$ Virology Laboratory and ${ }^{e}$ Department of Public \\ Health, Ministry of Health, Kuwait
}

Introduction and Objective: Malaria is endemic in more than 90 countries worldwide, and it is estimated that there are over 500 million clinical cases and 2.7 million deaths per year. In Kuwait, about 250 cases of malaria are detected every year by microscopy, which is very cumbersome and time-consuming. The aim of this study was to develop a rapid and specific diagnostic test to identify and treat individuals infected with malaria. Methods: About 250,000 immigrants come to Kuwait each year for residence or to work, and the majority of them come from developing coun- tries where malaria is endemic. A total of 200 blood samples were tested for malaria parasites by the OptiMAL method, and the results were compared with results obtained from thin and thick blood film microscopy. Results: During screening by microscopy it was found that $50 \%(100 / 200)$ of the blood samples were infected with malaria. Plasmodium vivax was detected in $80 \%(80 / 100)$ of the blood samples, whereas Plasmodium falciparum in $20 \%$ (20/100). Similarly, the OptiMAL test results indicated that $45 \%$ (90/200) of the blood samples were positive for malaria parasites. Infection with $P$. vivax was found in $81 \%$ (73/90) and $P$. falciparum in $18 \%(17 / 90)$ of blood samples, respectively. The microscopy method identified five more $P$. vivax in samples that were negative in the OptiMAL test. However, there was a good correlation between the results of microscopy and OptiMAL for the other 73 blood samples infected with $P$. vivax. The sensitivity of OptiMAL in detecting $P$. vivax was $40 \%$ when parasitemia was less than $100 / \mu 1$ of blood and rose to $100 \%$ when parasitemia was more than $100 / \mu 1$. Conclusions: The OptiMAL test may be as good as Giemsa's stained thick and thin blood films in the diagnosis of malaria in very remote areas where proper laboratory facilities and welltrained technicians are not available. The OptiMAL test is very simple, and the results usually available within $5 \mathrm{~min}$. 